\title{
RF MEMS BASED ON EPOXY-CORE CONDUCTORS
}

\author{
Yong-Kyu Yoon, Jin-Woo Park, and Mark G. Allen \\ School of Electrical and Computer Engineering \\ Georgia Institute of Technology \\ Atlanta, GA 30332
}

\begin{abstract}
In many RF MEMS applications, currents are confined to the outermost portions of conductors due to the skin effect. Conductors consisting of epoxy cores coated with metal are appropriate to consider for these applications, and in many instances are easier to fabricate than their solid-metal-core counterparts. As an example of this approach, this paper reports a three-dimensional, high-aspect-ratio, high Q-factor, solenoid-type $\mathrm{RF}$ inductor. The conductors of this inductor are fabricated using SU8 epoxy cores and subsequent metal electrodeposition. The SU8 core approach allows for relatively simple formation of extremely high aspect ratio columns for inductor sidewalls. In addition, an SU8 bridge fabrication technique has been realized using a double exposure and single develop scheme. A single, vialess metallization is then used to form the conducting paths of the inductor. By combining these techniques, epoxy-core RF inductors have been fabricated and tested. A single turn inductor that is $900 \mu \mathrm{m}$ in height and has a $600 \mu \mathrm{m}$ wide air-core area shows a maximum Q-factor of 84 and an inductance of $1.17 \mathrm{nH}$ at $2.6 \mathrm{GHz}$.
\end{abstract}

\section{INTRODUCTION}

Integrated 3-dimensional solenoid type RF inductors with high Q-factor fabricated using MEMS technology have been pursued for several years [1]. More recently, these devices have been integrated with CMOS circuits to form functional RF power amplifiers [2]. These devices are typically fabricated by plating through photoresist molds. However, the ratio of coil height to turn-to-turn pitch in many of these devices is relatively low due to difficulties in forming and filling extremely small, high aspect ratio via holes. The low profile coil suffers from magnetic flux leakage between relatively wide conductor line spaces, which can result in Q-factor decrease. Increased core height, leading to a geometry closer to an ideal solenoid, should yield more ideal performance. In addition, the previous inductors consisted of three discrete layers: lower electrode, via, and upper electrode. This construction not only leads to an increased number of electrodeposition steps, but also has the potential for increased via resistance and/or mechanical weakness at each interface.

In this paper, a tall solenoid inductor is proposed using SU8 epoxy as a core or backbone structure. This SU8 core is then covered in a single electrodeposition step. This approach differs from the conventional 3-D RF inductor fabrication, which uses via holes followed by via filling. With SU8 material, it is relatively easy to fabricate columns of a given aspect ratio as opposed to vias of the same aspect ratio [3]. In addition, metal electrodeposition on the column surface is much easier than via hole filling to obtain void-free and seamless conductor structures.

To fabricate inductors in a single metal deposition step, this column formation technique is combined with a double exposure and single development technique to form laterally-extended bridges on top of columns. Thus, SU8 column and bridge formation followed by metal coating is proposed as a method to realize inductors with high aspect ratio (up to 10:1) interconnect between lower and upper conductors. With this geometry, a large inductance in the same number of turns is obtained, and the device is geometrically closer to an ideal solenoid inductor.

It should be noted that due to the skin effect, as long as the metal coating is large in thickness compared with the skin depth at the operating frequencies of interest, no degradation of conductivity is expected in epoxy-core structures compared with metal-core structures. This approach is therefore also potentially of interest for other RF MEMS applications, such as transformers, switches and antennas.

\section{FABRICATION}

The fabrication process is described in Figure 1. In order to selectively coat metal only on the SU8 column, bridge, and bottom electrode definition area, three foundation layers are deposited on a glass substrate; a chromium (100nm thick) electrical contact layer for electroplating; a silicon dioxide (1000nm thick) passivation layer; and a titanium (Ti) / copper $(\mathrm{Cu}) /$ titanium (Ti) $(30 \mathrm{~nm} /$ $1000 \mathrm{~nm} / 30 \mathrm{~nm}$ ) sacrificial layer for selective seed layer definition. $\mathrm{The} \mathrm{Ti} / \mathrm{Cu} / \mathrm{Ti}$ and silicon dioxide layers are patterned for bottom electrode definition (Figure 1.1). A single SU8 layer is coated and soft baked, where the thickness of the SU8 layer becomes height of the inductor. A large optical dose is applied through the column mask and post-exposure baked at $95^{\circ} \mathrm{C}$ on a hot plate (Figure 1.2). Prior to development, a small optical dose is applied through the lateral bridge mask, and the structure is post-exposure baked at $95^{\circ} \mathrm{C}$ in an oven. After this two-step exposure and bake, the SU8 has the desired top bridge portion cross-linked at the top of the cross-linked columns (Figure 1.3). Developing the SU8 leaves the column and bridge. After curing the released structure in a $100^{\circ} \mathrm{C}$ oven, chromium/gold $(20 \mathrm{~nm} / 150 \mathrm{~nm})$ layers are coated using DC sputtering as the seed layer for subsequent electroplating (Figure 1.4). Removal of the previously-deposited copper and titanium layers leaves the $\mathrm{Cr} / \mathrm{Au}$ seed layers only on the surface of the SU8 structure and the bottom electrode area. Copper/gold layers are then electroplated in sequence to a thickness of 10-15 microns (Figure 1.5). Removal of the passivation silicon dioxide and initial chromium layer in sequence isolates the structure to complete the process (Figure 1.6). Unlike the SU8 multi-exposure-singledevelop technique described in [4], which utilizes anti-reflection coatings in order to control the thickness of the upper portion of the device, the technique described in this paper relies on control of the baking steps to achieve the final structure.

\section{EXPERIMENT AND RESULTS}

Fabricated SU-8 core structures prior to metal deposition and after metal coating to form solenoid inductors are shown in Figure 2 upper and lower, respectively. The height of the inductors is approximately 0.5 millimeter and copper and gold are electrodeposited in sequence in the thickness of $14 \mu \mathrm{m}$ and $1 \mu \mathrm{m}$, respectively, in order to form electrical inductor path. Two singleturn inductors, one $500 \mu \mathrm{m}$ tall with a $600 \mu \mathrm{m}$ core width, and one $900 \mu \mathrm{m}$ tall and $600 \mu \mathrm{m}$ in core width, are shown in Figure 3. They 
have been RF tested, and the obtained inductance and Q-factor as a function of frequency is shown in Figure 4. The $900 \mu \mathrm{m}$ device shows an inductance and a maximum Q-factor of $1.17 \mathrm{nH}$ and 84 (at $2.6 \mathrm{GHz}$ ), respectively, while the $500 \mu \mathrm{m}$ device shows an inductance and a maximum Q-factor of $0.77 \mathrm{nH}$ and 85 (at 2.5 $\mathrm{GHz}$ ), respectively. The relatively large Q-factors indicate that the RF performance of these devices is not degraded by conductors with epoxy cores compared with solid cores.

\section{CONCLUSION}

Epoxy-core fabrication approaches for RF MEMS conductors have been demonstrated. The fabrication advantages of this approach have been utilized to create tall, 3-D solenoid inductors. As predicted by the skin effect phenomenon, high Q-factor is obtained even though the interior core of the devices is nonconducting. This approach may also be of interest for other RF MEMS applications, such as transformers, switches and antennas.

\section{ACKNOWLEDGEMENT}

The authors would like to thank Mr. S.-W. Yoon and Prof. J. Laskar of Georgia Tech for their assistance with device measurement, and Mr. Y.-H. Joung and Mr. F. Cros of Georgia Tech for helpful technical discussion. Travel support has been generously provided by the Transducers Research Foundation and by the DARPA MEMS and DARPA BioFlips programs.

\section{REFERENCES}

1. Y.J.Kim and M.G.Allen, "Surface Micromachined Solenoid Inductors for High Frequency Applications," IEEE Trans. CPMT, Part C, vol. 21, No. 1, pp. 26-33, Jan. 1998.

2. Y.K.Yoon, E.Chen, M.G.Allen, and J.Laskar, "Embedded Solenoid Inductors for RF CMOS Power Amplifier," Proceeding of Solid-State and Actuators, Transducers'01 EurosensorsXV, Vol. 2, pp. 1114-1117, 2001

3. H.Lorenz, M.Despont, N.Fahrni, J.Brugger, P.Vettiger, P. Renaud, "High aspect ratio, ultrathick, negative-tone near-UV photoresist and its applications for MEMS," Sensors and Actuators, Part A, vol. 64, pp-33-39, 1998

4. F.G.Tseng, Y.J.Chuang, and W.K.Lin, "A Novel Fabrication Method of Embedded Micro Channels Employing Simple UV Dosage control and Antireflection Coating", Proceedings of IEEE MEMS 2002, Las Vegas, pp. 69-72, 2002

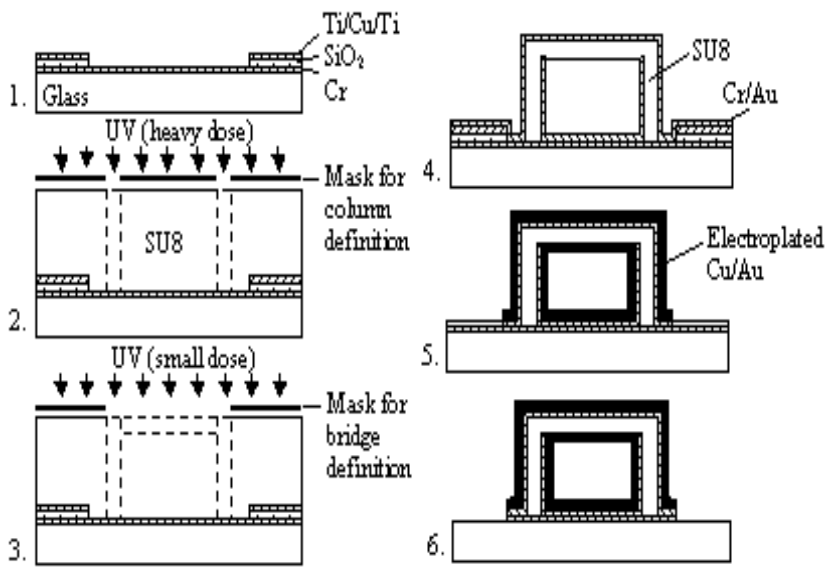

Figure 1. Fabrication process

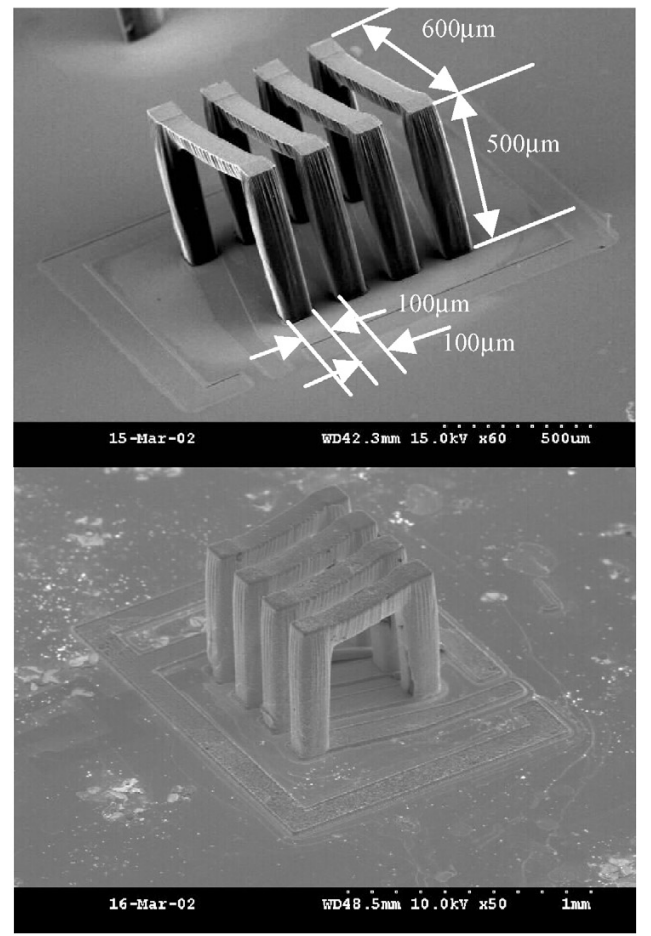

Figure 2. (Upper) Fabricated SU-8 core structures prior to metal deposition. (Lower) After metal coating to form solenoid inductors. Note that the height of the inductors is approximately 0.5 millimeter.
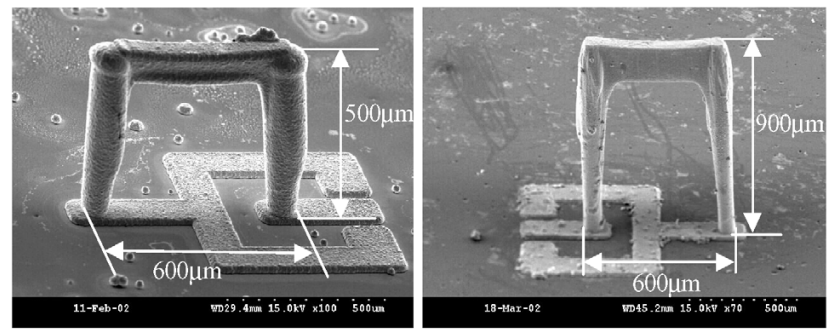

Figure 3. Single turn inductors; (Left) $500 \mu \mathrm{m}$ device, (Right) $900 \mu \mathrm{m}$ device.

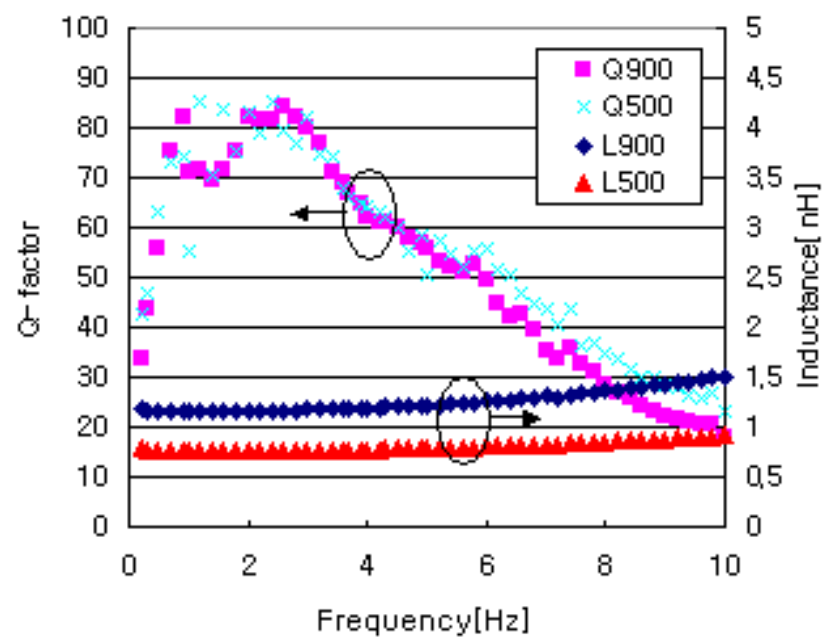

Figure 4. Inductance and $Q$-factor for two single turn inductors. 